\title{
Reconstruction of the solar EUV irradiance from 1996 to 2010 based on SOHO/EIT images
}

\author{
Margit Haberreiter ${ }^{1, *}$, Véronique Delouille ${ }^{2}$, Benjamin Mampaey $^{2}$, Cis Verbeeck ${ }^{2}$, Giulio Del Zanna ${ }^{3}$ \\ and Seth Wieman ${ }^{4}$ \\ 1 Physikalisch-Meteorologisches Observatorium and World Radiation Center, Dorfstrasse 33, CH-7260 Davos Dorf, Switzerland \\ *Corresponding author: margit.haberreiter@pmodwrc.ch \\ 2 STCE/Royal Observatory of Belgium, Avenue Circulaire 3, 1180 Brussels, Belgium \\ 3 DAMTP, Centre for Mathematical Sciences, Wilberforce Road, Cambridge CB3 0WA, UK \\ 4 University of Southern California Space Sciences Center, 835 Bloom Walk, Los Angeles, CA 90089, USA
}

Received 10 October 2013 / Accepted 28 August 2014

\begin{abstract}
The solar Extreme UltraViolet (EUV) spectrum has important effects on the Earth's upper atmosphere. For a detailed investigation of these effects it is important to have a consistent data series of the EUV spectral irradiance available. We present a reconstruction of the solar EUV irradiance based on SOHO/EIT images, along with synthetic spectra calculated using different coronal features which represent the brightness variation of the solar atmosphere. The EIT images are segmented with the SPoCA2 tool which separates the features based on a fixed brightness classification scheme. With the SOLMOD code we then calculate intensity spectra for the 10-100 $\mathrm{nm}$ wavelength range and each of the coronal features. Weighting the intensity spectra with the area covered by each of the features yields the temporal variation of the EUV spectrum. The reconstructed spectrum is then validated against the spectral irradiance as observed with SOHO/SEM. Our approach leads to good agreement between the reconstructed and the observed spectral irradiance. This study is an important step toward understanding variations in the solar EUV spectrum and ultimately its effect on the Earth's upper atmosphere.
\end{abstract}

Key words. Corona - Sun - Solar cycle - Spectral irradiance - Space Weather

\section{Introduction}

The solar Extreme UltraViolet (EUV) spectrum, which covers the wavelength range from 1 to $120 \mathrm{~nm}$, varies on timescales of minutes to days, the 27-day solar rotation period, and the 11-year solar cycle. The peculiar 2008-2009 solar minimum also gave rise to the question whether a long-term trend can be observed, both in the EUV emission of the Sun and in the parameters of the Earth's upper atmosphere. Whether the long-term trend is positive or negative is still a topic of current debate (e.g., Lean et al. 2011a; Laštovička 2013). At the same time the variability of the EUV spectrum has considerable impact on the upper Earth's atmosphere, i.e., on the density, temperature, and total electron content (see e.g. Qian \& Solomon 2012), and is an important driver for space weather (Lilensten et al. 2008).

A detailed understanding of the EUV variability requires consistent long-term observations from space. However, the EUV datasets - similar to other solar irradiance datasets are typically inhomogeneous, as they are obtained from different instruments with different temporal and spectral resolution. In addition, all space instruments are subject to degradation, which makes the gathering of a homogeneous time series more challenging. The degradation of current EUV instruments in space has been discussed in detail by BenMoussa et al. (2013).

Several approaches have already been employed to model solar variations in the EUV. Using the NRL model, Warren et al. (2001) calculated optically thin intensities for coronal holes, the quiet Sun, and active regions based on the emission measure distributions determined from spectrally and spatially resolved observations. They obtained good agreement with the rotational variability of observations from the Atmospheric Explorer (Hinteregger et al. 1981). Furthermore, Kretzschmar et al. (2006) employ an empirical approach which is based on a number of specific spectral lines and investigate to which extent specific spectral lines can be used to determine the variation of the EUV spectrum. Similarly, Dudok de Wit \& Bruinsma (2011) and Dudok de Wit et al. (2014) are very successful in reproducing the EUV spectrum using different sets of proxy data, while Cessateur et al. $(2011,2012)$ employed SSI observations in selected passbands. Moreover, Lean et al. (2011b) developed empirical models of the EUV irradiance variability using multiple regression of the $\mathrm{Mg}$ II and F10.7 solar activity indices along with irradiance observations of the descending phase of solar cycle 23, which also leads to good agreement with the observations. Fontenla et al. (2011) present a set of updated models of the chromosphere, the transition region and corona, and reconstruct the EUV variability based on the segmentation of images taken with the Atmospheric Imaging Assembly (AIA; Lemen et al. 2012) onboard the NASA Mission Solar Dynamics Observatory (SDO). They obtained an agreement within the uncertainty range with observations from the Solar EUV Experiment (SEE; Woods et al. 2005) onboard the NASA Thermosphere Ionosphere Mesosphere Energetics and Dynamics (TIMED) mission. More recently, Thuillier et al. (2014) carried out a reconstruction of solar irradiance in various spectral bands over the solar spectrum, including the EUV as observed with the 
Table 1. Solar features employed in the spectral synthesis, listed increasing with brightness.

\begin{tabular}{|c|c|c|c|c|c|c|}
\hline Class & 1 & 2 & 3 & 4 & 5 & 6 \\
\hline Solar & Coronal & Quiet & Quiet & Active & Active & Active \\
\hline Feature & Hole & Sun 1 & Sun 2 & Region 1 & Region 2 & Region 3 \\
\hline Label & $\mathrm{CH}$ & QS1 & QS2 & AR1 & AR2 & AR3 \\
\hline$C_{171}$ & 0.11 & 0.23 & 1.00 & 2.78 & 5.21 & 7.92 \\
\hline$C_{195}$ & 0.12 & 0.24 & 1.00 & 2.77 & 5.47 & 9.37 \\
\hline
\end{tabular}

SolACES instrument (Schmidtke et al. 2006a) onboard the ISS/SOLAR platform (Schmidtke et al. 2006b). For the EUV reconstruction the authors employ the SOLMOD code (Haberreiter 2011) along with the segmentation of SDO/AIA images and find a reasonable agreement with the SolACES irradiance measurements.

Our approach in this paper takes advantage of more than 16 years of EUV irradiance and EUV telescope measurements taken onboard the SOlar and Heliospheric physics Observatory (SOHO). Our analysis is based on the segmentation of images taken in the $171 \AA$ and $195 \AA$ channels with the Extreme UV Imaging Telescope (EIT; Delaboudinière et al. 1995). Using the Spatial Possibilistic Clustering Algorithm (SPoCA2) tool described in Verbeeck et al. (2014), these images are segmented into six different features. This dataset of EIT images covers approximately one and a half solar cycles, including two solar minima. During the same time period, the SOHO/SEM instrument (Didkovsky et al. 2010) has provided excellent EUV irradiance data, which are used for validation. These extended datasets are extremely valuable for constraining the reconstruction models over all solar activity phases, in particular over one solar cycle. This is an important step toward understanding the causes of any potential long-term trend of Solar Spectral Irradiance (SSI) in the EUV.

The remainder of the paper is structured as follows. In Section 2 the SEM data will be presented. Then, in Section 3 the spectral synthesis code SOLMOD is discussed. In Section 4 the procedure to process the EIT images and the details of the SPoCA2 tool are described in detail. Finally, we present the results in Section 5 and the conclusions in Section 7.

\section{SOHO/SEM irradiance data}

The Solar EUV Monitor (SEM; Judge et al. 1998), part of the Sun Charge, Element, and Isotope Analysis System (CELIAS; Hovestadt et al. 1995) onboard the SOHO mission, is a highly stable grating spectrometer that has provided nearly continuous absolute EUV irradiance measurements in the 26-34 nm and 0.1-50 nm bandpasses since January 1996. The only interruption was seen in the summer of 1998. The instrument uses a freestanding transmission grating and silicon photodiode detectors that were developed for long-term stability (Ogawa et al. 1993). The SEM instrument sensitivity was determined prior to launch from calibrations (McMullin et al. 2002) at the National Institute of Standards and Technology Synchrotron Ultraviolet Radiation Facility (NIST/SURF). Instrument calibration has been maintained based on an ongoing series of sounding rocket measurements using a clone of the SEM instrument that is calibrated prior to and following each sounding rocket flight, as well as a neon Rare Gas Ionization Cell (RGIC) absolute detector. These calibration rocket flights have shown that the SEM instrument has experienced some loss of sensitivity which is attributed to the build-up and subsequent polymerization of hydrocarbon contaminants by solar UV radiation. This change in sensitivity has been corrected based on a time and wavelength dependent degradation model. Current (Version 3.1) calibrated irradiance measurements from the on-orbit SEM are in agreement with all sounding rocket measurements (which includes a total of eight sounding rocket flights between 1996 and 2012) to within about 5\% (Didkovsky et al. 2010; Wieman et al. 2011).

Recent refinements to the SEM data processing algorithm (Wieman et al. 2014) for the period of overlap between the SOHO and SDO missions have resulted in calibrated SEM irradiance values which are in better agreement with those from SDO/EVE (Woods et al. 2012). The most pronounced effect of these refinements is a systematic reduction of approximately $20 \%$ in the values of the SEM 26-34 nm irradiance compared to the SEM Version 3.1 values. While a new release of the SEM data based on this revised data processing approach is imminent, we compare the SOLMOD reconstruction with the Version 3.1 SEM irradiances as they are, at the time of the present work, the most recent available which cover the entire SOHO mission dating back to 1996.

\section{Spectral synthesis with SOLMOD}

As a starting point for the reconstruction of the solar EUV irradiance we use the approach by Fontenla et al. $(2009,2011)$ also pursued by Haberreiter $(2011,2012)$. Synthetic solar intensity spectra are calculated with the Solar Modelling (SOLMOD) code (Haberreiter, 2011). The atomic dataset in SOLMOD is based on the CHIANTI database (Dere et al. 1997; Landi et al. 2006) including the update of the forthcoming CHIANTI V8. We calculate spectra for five components of the intensity distribution of the solar corona: quiet corona (QS1), coronal quiet network (QS2), coronal active network (AR1), active regions (AR2), and bright active regions (AR3). A sixth class is defined for coronal holes. Its intensity is calculated with a fixed contrast of $C_{\mathrm{CH}}=0.5$ with respect to the quiet corona (QS1); see Table 1 for the list of the coronal features used to model the solar EUV variability. Also listed are the theoretical contrasts $C_{171}$ and $C_{195}$ as obtained from the spectral synthesis calculation for the EIT $171 \AA$ and $195 \AA$ channels as described below.

The synthetic calculations are based on a set of models that represent the temperature variation of the optically thin corona as described by Haberreiter (2011). For the coronal emission we account for solar species with ionization stages two and higher. The level populations are calculated with an optically thin approach, i.e., the radiation field is neglected in the calculation of the statistical equation. SOLMOD calculates the emergent spectrum in spherical symmetry which allows the emission beyond the solar limb to be realistically calculated (for details see, e.g., Haberreiter et al. 2008; Thuillier et al. 2011). This is important for the extended corona, in particular 
M. Haberreiter et al.: Reconstruction of the solar EUV irradiance from 1996 to 2010
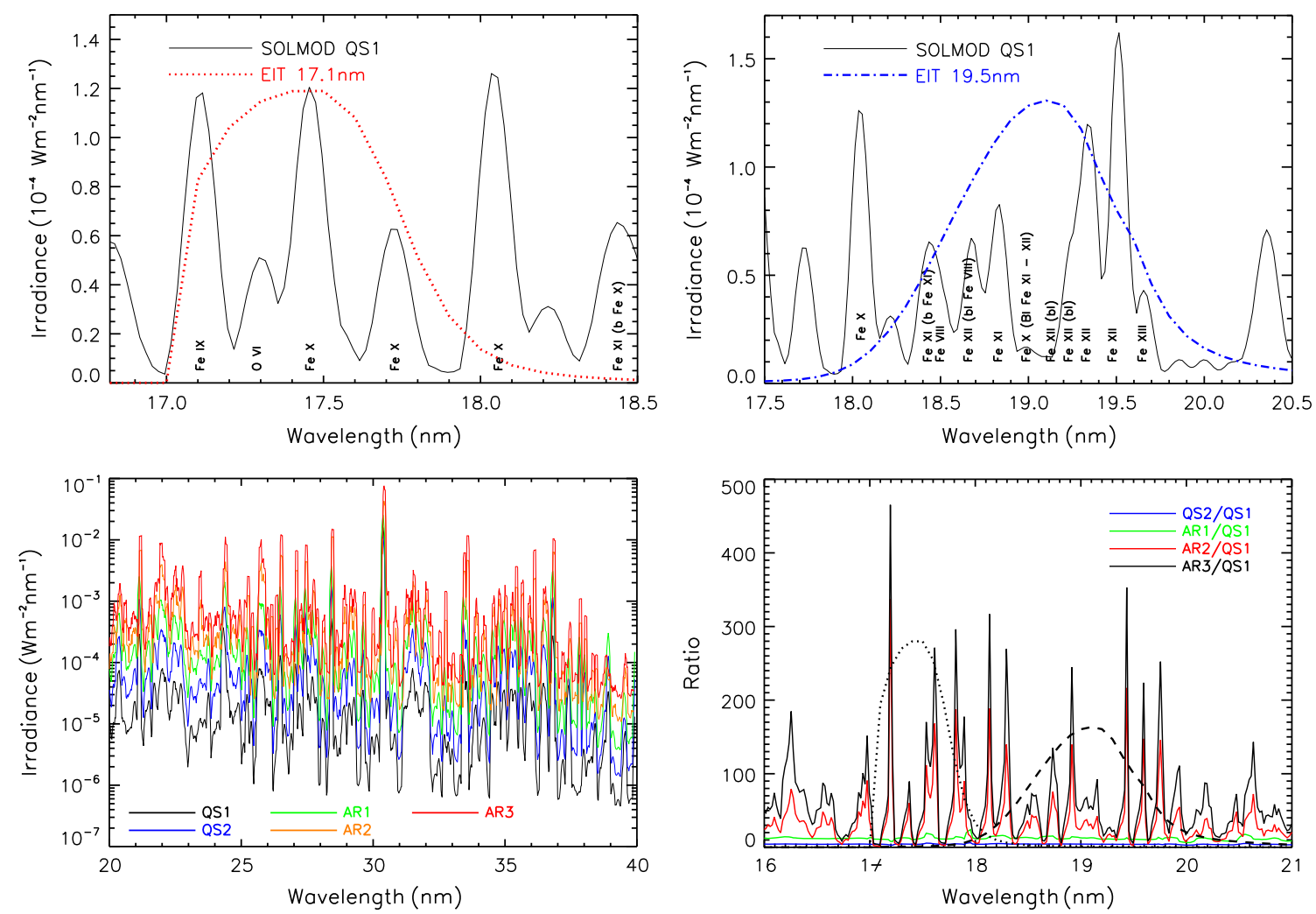

Fig. 1. Top panels: synthetic EUV irradiance spectrum (black line) convolved with a 1- $\AA$ boxcar calculated for the feature QS1, along with the passbands of the EIT $171 \AA$ (left panel, red dotted line) and $195 \AA$ (right panel, blue dashed line) channels (in arbitrary scale). Bottom panels: the left panel shows the irradiance spectra for QS1 (black), QS2 (blue), AR1 (green), AR2 (orange), and AR3 (red). The right panel gives the ratios of the synthetic spectra for each component to the QS1 spectrum. The EIT $171 \AA$ and $195 \AA$ passbands are indicated again.

to account for limb brightening, the increase of the coronal emission at the limb. The top right panel of Figure 1 shows the spectrum as calculated with SOLMOD around the EIT $171 \AA$ and $195 \AA$ passbands (dashed blue and dotted red lines, respectively). The bottom right panel shows the ratio of the different spectra with respect to the QS2 spectrum. From this plot it is clear that not all spectra contribute in the same way to the irradiance variation in the EUV.

Applying the EIT passbands to the synthetic spectra provides us with theoretical irradiance values for each coronal feature, shown in Figure 2. The values are normalized to QS2 and given in Table 1. These values are the basis for the segmentation as described in Section 4 and shown in the bottom right panel.

The time-dependent contribution of each of the spectra is determined from the segmentation analysis using the SPoCA2 tool as described in the following section. We note that for the current study we examine the pure coronal EUV variation through the detailed image segmentation, and leave the position-dependent background radiation from the chromosphere and transition region to an upcoming publication.

\section{Image segmentation}

\subsection{Selection of EIT dataset}

The image segmentation presented here relies on images taken with the SOHO/EIT instrument in the $171 \AA$ and $195 \AA$ channels over a time span of over 16 years. In particular, for the present study, we analyze images from 4 March 1996 to

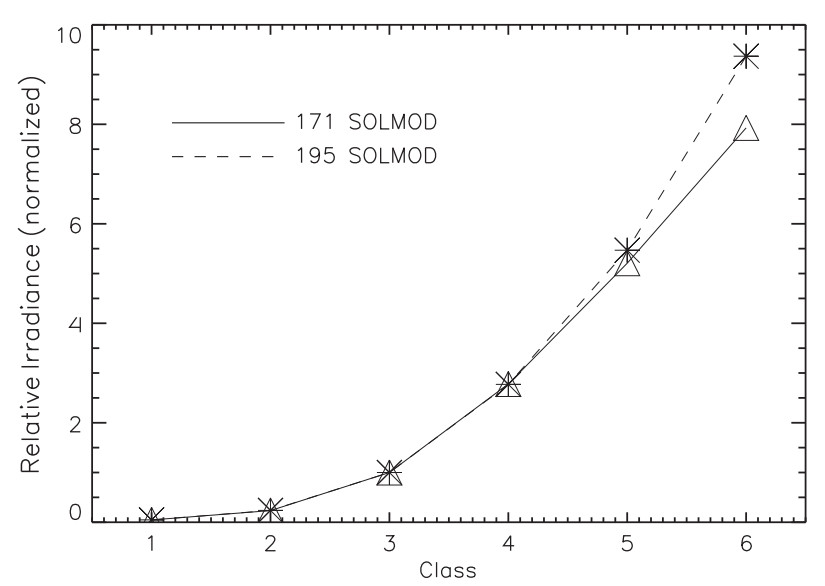

Fig. 2. Synthetic contrast calculated from the irradiance spectra for the EIT $171 \AA$ (solid line with triangles) and $195 \AA$ (dashed line with stars) passbands.

29 February 2012. The EIT images for this time frame are provided with a degradation correction. The degradation correction is described in detail by BenMoussa et al. (2013) and Clette et al. (2002). In short, the approach is that for each period between two successive EIT bake-outs, the EIT integrated fluxes are correlated with the Mg II index and detrended using a linear fit. In order to adequately process the EIT archive, we first build a homogeneous synoptic dataset from the set of images which have a science objective FITS header keyword of the type FULL SUN. 
To exclude partial data we selected images with no missing blocks. Only images with a shutter close time between 2 and $5.5 \mathrm{~s}$ were kept. These three criteria based on FITS keywords are, however, not sufficient to eliminate all corrupted images, such as images suffering from a proton storm. To remove these, statistics concerning the pixels intensities located on the solar disk were computed and outliers removed from the dataset. In addition, a list of corrupted images was kindly provided by the current Principal Investigator of the EIT instrument, F. Auchère (private communication).

The resulting datasets contain 155083 EIT FITS files for the $171 \AA$ bandpass and 15055 FITS files for the $195 \AA$ bandpass. For each wavelength, about $3 \%$ of the images are $512 \times 512$ in size and the remaining $97 \%$ are $1024 \times 1024$. In order to provide a uniform dataset we decided to remap the $512 \times 512$ onto $1024 \times 1024$ images. One pixel in a $512 \times 512$ image becomes four pixels in the corresponding $1024 \times 1024$ image; each of these four pixels receiving one fourth of the pixel intensity from the original image. The resulting dataset spans the period March 1996-February 2012 at a typical cadence of four images per day and per bandpass.

Two more transforms are performed on this dataset. First, since the calibration procedure for EIT does not rotate the images to align the solar north upward, we use a cubic interpolation procedure to rotate the images so that they are all aligned. This allows for easier visualization of the results. Second, we are interested in a segmentation up to $1.4 R_{\odot}$, where $R_{\odot}$ is the solar radius. A limb brightness correction is therefore needed and is applied on the eit-prepped images as described in Verbeeck et al. (2014). Note that the spectral synthesis code SOLMOD accounts for the limb brightening naturally due to the lineof-sight integration carried out in spherical symmetry. In the preparation stage before the analysis, we also remove pixels from the EIT images whose intensity is smaller or equal to $2 \mathrm{DN} / \mathrm{s}$ from the computation of area coverages in order to avoid counting pixels that are essentially read-out noise. In the $171 \AA$ bandpass, there is no pixel located within the $1.4 R_{\odot}$ with a value below $2 D N / s$. In the $195 \AA$ bandpass, about $0.42 \%$ of the total number of pixels within $1.4 R_{\odot}$ have a value below $2 \mathrm{DN} / \mathrm{s}$ and are found to be beyond $1.15 R_{\odot}$. These pixels are removed from the analysis. In the work presented here, the images are not corrected for any scattered light.

\subsection{SPoCA2 fuzzy clustering algorithm}

To perform EUV irradiance reconstruction at a given time, the proportions of the Sun covered by the six coronal features must be known. In the terminology of image segmentation, we call these features "classes". Such proportions can be derived via a pixel intensity-based segmentation of an EUV images. The pixels with high intensities correspond to active regions, those with intermediate values to quiet-Sun areas, and the lowest intensity pixels can be attributed to coronal holes. Due to the difference in emission and temperature of these structures, it is expected that these classes will naturally form clusters in the space of pixel intensity values. In the general case, we consider $C$ classes to be distinguished.

In this paper we use the SPoCA2 fuzzy clustering approach. We recall here the formula to be used in the present context. The reader is referred to Verbeeck et al. (2014) for a complete description. In fuzzy clustering, every pixel obtains a membership value to each of the $C$ classes via the minimization of an intra-cluster variance. Such minimization is reached through an iterative algorithm; see Eq. (1). During the initialization step (described in Sect. 4.3 below) the values for the class centers, i.e., the center intensity value for a certain class, are determined. Next, every subsequent iteration consists of two steps: first, the membership value of every pixel to each class is calculated; second, for every class the intensity value for the class center is updated. These steps are repeated until convergence of the class centers sets in, the result being a final membership value of every pixel to every class. To obtain a crisp segmentation, we assign every pixel to exactly one class via some decision rule. Here, we use the rule that assigns the pixel to the class for which its membership value is highest (see also Sect. 4.4).

More formally, let $N$ be the number of pixels in each image and let $x_{j} \in \mathbb{R}$ be the pixel intensity value observed in one EUV channel at a particular location of the Sun. A fuzzy clustering algorithm searches for $C$ different compact clusters amongst the $x_{j}$ 's in the set $X=\left\{x_{j} \in \mathbb{R} \mid 1 \leq j \leq N\right\}$ of all pixel intensity values. It does so by computing both a fuzzy partition matrix $U=\left(u_{i j}\right)_{1 \leq i \leq C, 1 \leq j \leq N}$ and the cluster centers $B=\left\{b_{i} \in \mathbb{R} \mid 1 \leq i \leq C\right\}$. The scalar $u_{i j}=u_{i}\left(x_{j}\right) \in[0,1]$ is called the membership value of $x_{j}$ to class $i$ (Bezdek 1981).

Our algorithm is based on the Possibilistic C-means algorithms (Krishnapuram \& Keller 1993, 1996), whose aim is to minimize a regularized fuzzy intra-class variance. We use a slightly modified version of the original PCM algorithm that we call PCM2:

$$
u_{i j}=\left[1+\left(d^{2}\left(x_{j}, b_{i}\right) / \eta_{i}\right)^{2}\right]^{-1} \text { and } b_{i}=\sum_{j=1}^{N} u_{i j}^{2} x_{j} / \sum_{j=1}^{N} u_{i j}^{2}
$$

where $d^{2}(\cdot, \cdot)$ indicates Euclidian distance and where the regularization parameter $\eta_{i}$ is computed as the intra-class dispersion:

$$
\eta_{i}=\frac{\sum_{j=1}^{N} u_{i j}^{2} d^{2}\left(x_{j}, b_{i}\right)}{\sum_{j=1}^{N} u_{i j}^{2}}
$$

The above PCM2 method proceeds on a pixel-per-pixel basis. EIT images are however routinely affected by cosmic rays. They generate high intensity pixels which are uncorrelated with neighboring pixel values. To mitigate this effect, we add a spatial regularization term in the objective function to be minimized (Barra et al. 2008). This forces memberships in a neighborhood to be as close as possible. This is formally described as follows.

Let $\mathscr{N}_{j}$ denote the neighborhood of pixel $j$, Card $\left(\mathscr{N}_{j}\right)$ be the number of elements in $\mathscr{N}_{j}$, and $\mathscr{N}^{j}=\mathscr{N}_{j} \cup\{j\}$. The Spatial Possibilistic Clustering Algorithm (SPoCA2) finds the membership map $U$ and centers $B$ through the following iterations:

$$
\begin{aligned}
u_{i j} & =\left[1+\left(\frac{\sum_{k \in \mathcal{N}^{j}} \beta_{k} d^{2}\left(x_{k}, b_{i}\right)}{\eta_{i}}\right)^{2}\right]^{-1} \text { and } \\
b_{i} & =\frac{\sum_{j=1}^{N} u_{i j}^{2} \sum_{k \in \mathcal{N}^{j}} \beta_{k} x_{k}}{2 \sum_{j=1}^{N} u_{i j}^{2}},
\end{aligned}
$$



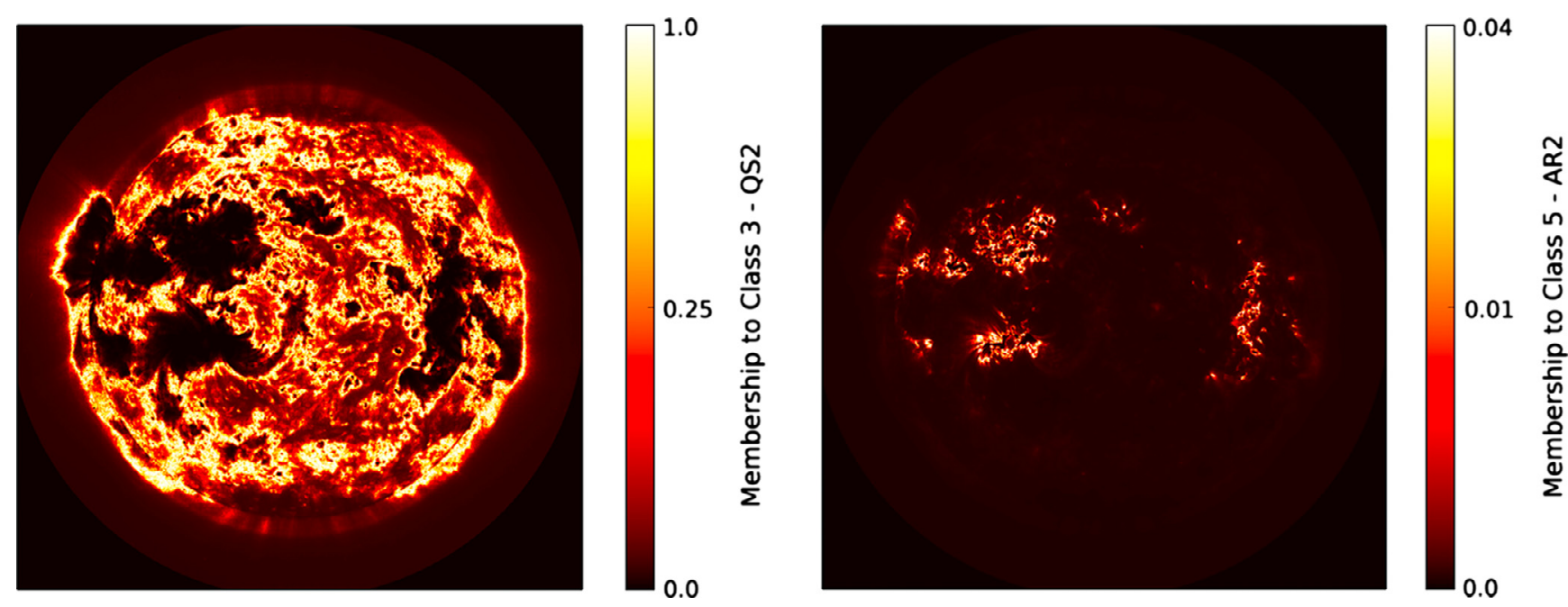

Fig. 3. Maps of the membership values for the coronal quiet network (QS2, left panel) and active regions (AR2, right panel) corresponding to a 171 Å image taken on 23 November 2003.

where the weight $\beta_{k}$ are defined as:

$$
\beta_{k}=\left\{\begin{array}{cc}
1 & \text { if } k=j \\
\frac{1}{\operatorname{Card}\left(\mathscr{N}_{j}\right)} & \text { otherwise }
\end{array}\right.
$$

The membership value $u_{i j}$ in Eq. (1) now depends on the distance between all $x_{j} \in \mathscr{N}^{j}$ and thus regularizes the memberships of pixel $j$ with respect to all of his neighbors $\mathscr{N}_{j}$. We use a $3 \times 3$ pixel neighborhood around each pixel. The iteration described by Eq. (1) requires an initialization, which is typically performed through one iteration of the Fuzzy C-Means algorithm (see Verbeeck et al. 2014, for more details).

Figure 3 displays an example of a membership map for the coronal quiet network (QS2) and for the active region (AR2). Membership values for QS2 are high near active regions and decrease with increasing distance from the AR, where the QS1 class is more prominent. The AR2 class has large membership values for coronal loops and these values decrease as the coronal loops become fainter. The central, i.e., hottest part, of the AR will have a low membership with respect to class AR2 and a large one with respect to class AR3.

Several rules can be devised to convert fuzzy membership values to crisp segmentation, as is detailed in Verbeeck et al. (2014). In this work, we use the Maximum rule: given the membership maps $U$ and centers $B$, the segmented maps are obtained by assigning a pixel $j$ to the class $c$ for which it has the maximum membership value: $u_{c j}=\max _{i \in\{1, \ldots, C\}} u_{i j}$.

\subsection{Determination of class centers}

In order to choose the center to be taken as a reference, we perform a pre-study on a dataset consisting of one image per bandpass every 14 days during 11 years, from 1 January 1998 until 31 December 2008, i.e., during one full solar cycle. On this dataset, the SPoCA2 algorithm described in Eq. (1) is applied on a frame-by-frame basis. The corresponding values for the class centers are shown in Figure 4. As expected, all center values exhibit a solar cycle dependence. We note that this solar cycle variation of the class center values is - in the current approach - not taken into account in the reconstruction, as only the area coverage of each of the classes determines the solar cycle variation. However, we intend to study this in further detail in future studies. The third class represents the coronal quiet network. It shows some stability over time and is also the most predominant in terms of solar surface coverage. For these reasons, we decided to take this third class as the reference class. We then calculated the irradiance ratio for the EIT $171 \AA$ and $195 \AA$ passbands using the SOLMOD code. Figure 2 gives the SOLMOD contrast values relative to the third class. For consistency, these contrast values were then used relative to the third class (computed with SPoCA2) in order to compute the absolute intensity values (in $\mathrm{DN} / \mathrm{s}$ ) for the other five class centers. Table 2 provides these class centers values, which are further used to process the homogeneous EIT dataset described in Section 4.1. The values based on the SOLMOD calculations, as given in Table 2, are also displayed in Figure 4 as red dotted lines.

In order to understand the strong increase of the area associated to AR1, AR2, and AR3 identified from the EIT images in 2011, we investigated the variation of the mean and total intensity of the on-disk pixels in the $171 \AA$ and $195 \AA$ channel. Figure 6 shows the mean (top panel) and total (bottom panel) for the $171 \AA$ and $195 \AA$ dataset. As it turns out, at the beginning of 2011, a strong increase in both the mean and total intensity is detected. One reason for this could be that the EIT calibration is less reliable in the later phase of the SOHO mission. As the SPoCA tool uses fixed intensity values for the identification of each coronal feature, an increase of the overall EIT intensity signal leads to an overestimation in the area associated to active regions and at the same time an underestimation of the quiet Sun features, leading to incorrect results in the reconstruction. Therefore, we (for now) exclude EIT data after 31 December 2010 from the reconstruction. The reconstruction time series will be extended to more recent times as soon as this issue is solved and newly calibrated images become available.

\subsection{Determination of area coverage}

Given the center values in Table 2, the membership maps $U$ are computed for the complete EIT dataset by attribution, that is, using the left-hand part of Eq. (1). Segmentation maps are then obtained by assigning a pixel $j$ to the class for which its membership values is highest. 

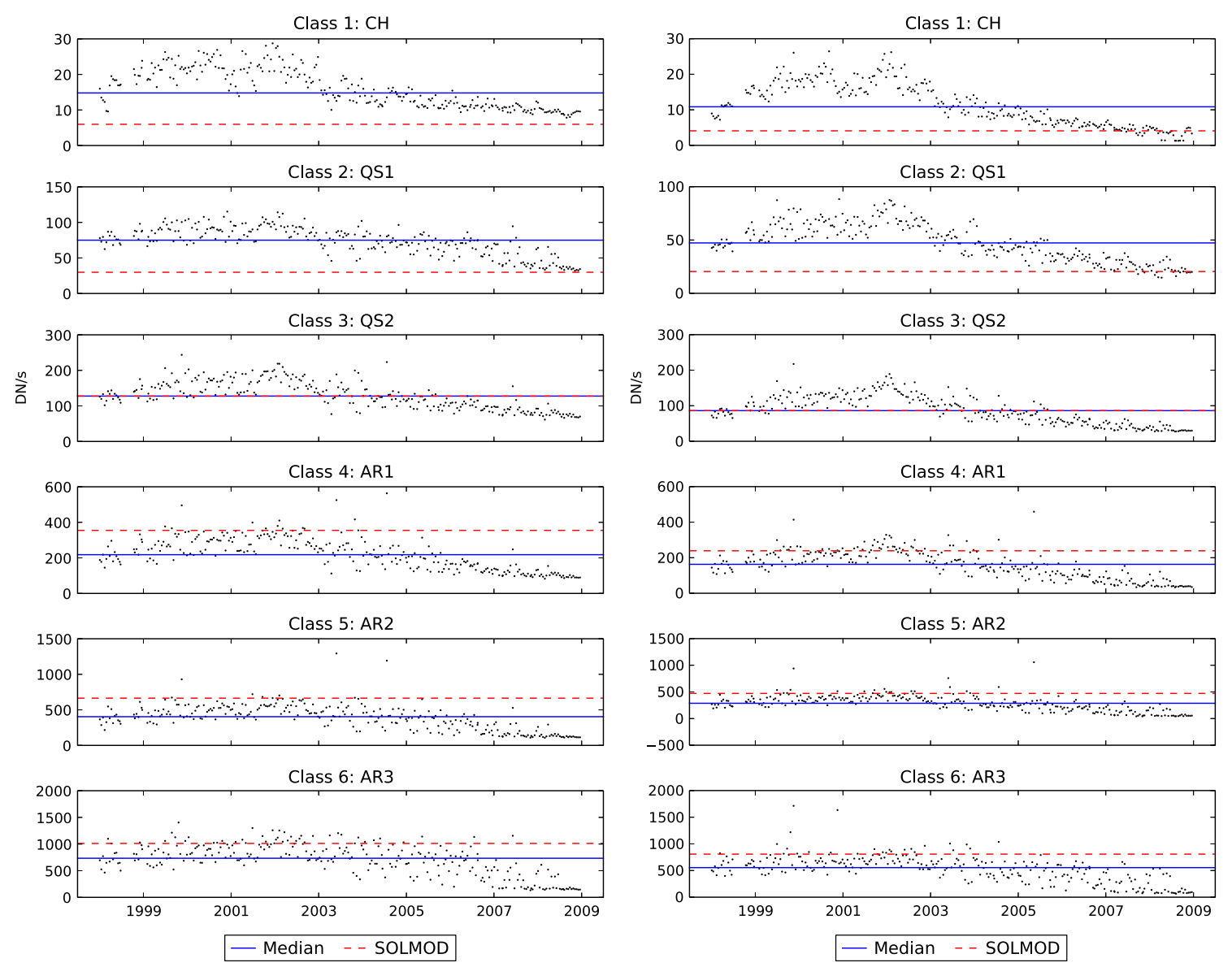

Fig. 4. Evolution of centers of classes computed with SPoCA2 on a frame-by-frame basis on EIT $171 \AA$ (left) and $195 \AA$ (right) images. The dataset consists of one image per bandpass every 14 days during the period 1 January 1998 until 31 December 2008. The blue solid lines represent the median value over the whole period, while the red dotted lines are the values found using the SOLMOD ratios.

Table 2. Values for class centers. The center of Class 3 (C3, in bold) is the median of class center values obtained by SPoCA2 in the pre-study dataset. The other class centers were determined from C3 and the SOLMOD contrast values, repeated here for convenience.

\begin{tabular}{llccrrr}
\hline \hline & $\mathrm{C} 1$ & $\mathrm{C} 2$ & $\mathrm{C} 3$ & $\mathrm{C} 4$ & $\mathrm{C} 5$ & $\mathrm{C} 6$ \\
\hline$I_{171}(\mathrm{DN} / s)$ & 6.0 & 29.9 & $\mathbf{1 2 7 . 6}$ & 354.7 & 664.5 & 1011.6 \\
$I_{193}(\mathrm{DN} / s)$ & 4.1 & 20.4 & $\mathbf{8 6 . 2}$ & 238.9 & 471.4 & 807.6 \\
$C_{171}$ & 0.11 & 0.23 & 1.00 & 2.78 & 5.21 & 7.92 \\
$C_{195}$ & 0.12 & 0.24 & 1.00 & 2.77 & 5.47 & 9.37 \\
\hline
\end{tabular}

The segmentation maps can then be summarized by computing the number of pixels belonging to a particular class, or area coverage. These are represented in Figure 5 for the 16 years of EIT archive when taking into account $95 \%$ of the solar radius, thus leaving out the solar limb. On the graphs in Figure 5, the height filled up by one color gives the area coverage for the corresponding class, with the six area coverages summing to one. The classes are displayed from bottom to top in order of increased intensity values, from the coronal holes (Class $1, \mathrm{CH})$ up to the brightest part of an active region (Class 6, AR3). The bottom panels of Figure 5 show a zoom-in of the $\mathrm{CH}$ class for each EIT channel, respectively. Both channels give different results for the area coverage of the coronal features. First, the $171 \AA$ channel is not appropriate to identify coronal holes. To compensate for this, we have added the $195 \AA$ dataset into our analysis, which provides a very good detection of the coronal hole area. Second, the area coverage factor for QS1 is significantly larger in the case of the $195 \AA$ channel and less so for the $171 \AA$ channel, and vice versa for QS2. Finally, the area coverage for AR1, AR2, and AR3 is comparable. Figure 5 shows the direct comparison of these area coverage identified with SPoCA2. We would like to emphasize that the detailed structure of the loops can be very complex, however, the most important parameter for irradiance reconstruction purposes is the line-of-sight integration through the plasma at different temperatures. Therefore the area on and beyond the solar disk covered by the coronal features, and as determined in this work, is the essential parameter driving the irradiance variations. It is clear that the highly complex coronal loop structures are not accounted for by this approach. As for the optically thin emission, it is the emitting volume of plasma, the approach presented here describes with sufficient accuracy the mean emission of the various features of the corona. 
M. Haberreiter et al.: Reconstruction of the solar EUV irradiance from 1996 to 2010
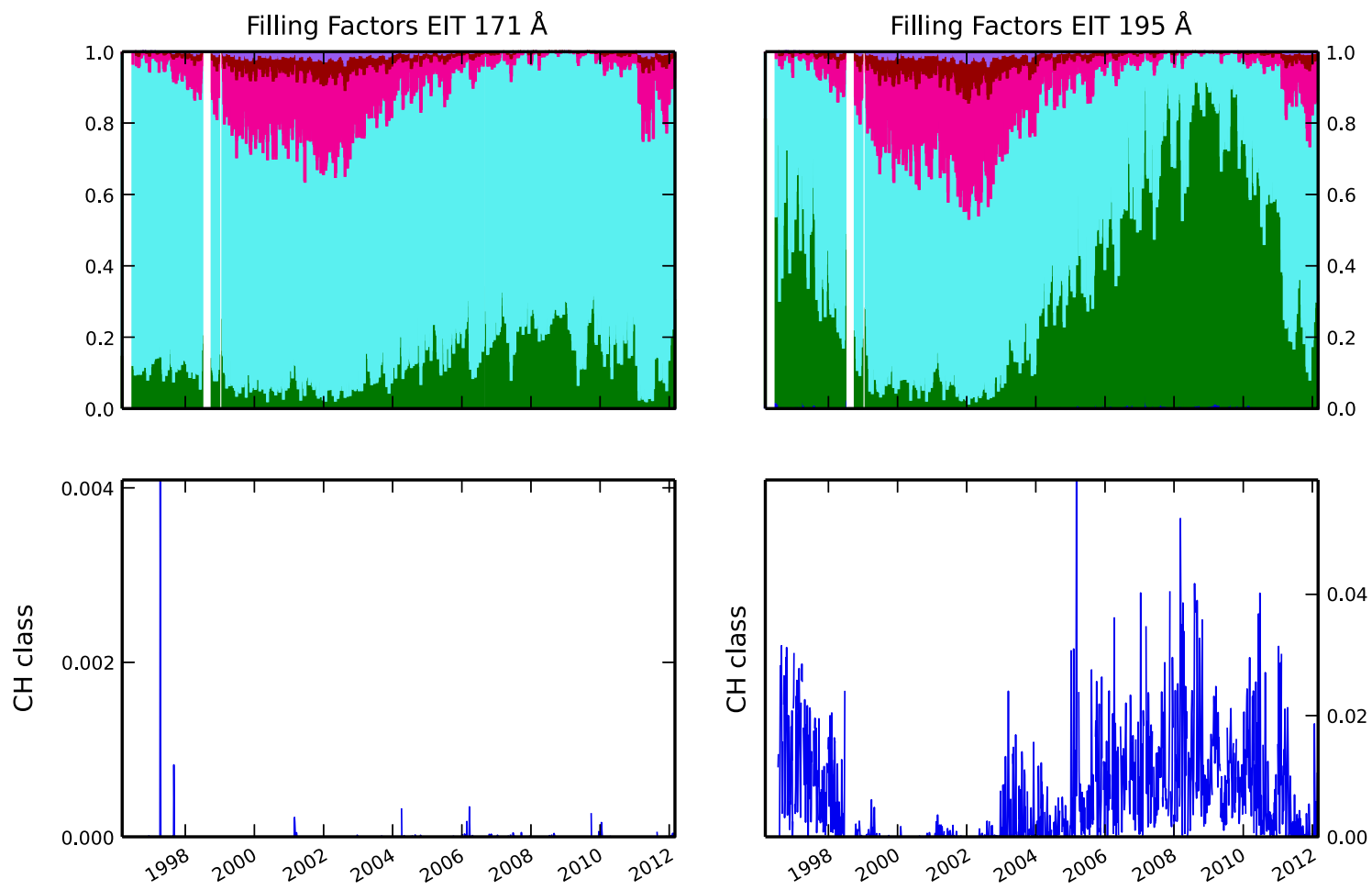

$$
\begin{array}{|lll|}
\hline \text { Class 1: } \mathrm{CH} & \text { Class 3: QS2 } & \text { Class 5: AR2 } \\
\text { Class 2: QS1 } & \text { Class 4: AR1 } & \text { Class 6: AR3 } \\
\hline
\end{array}
$$

Fig. 5. Area coverages for the six classes spanning the period March 1996-December 2012 for the EIT $171 \AA$ \&andpass (top left panel) and the EIT $195 \AA$ bandpass (top right panel). The extent on the $y$-axis gives the area coverage for the corresponding class, with the six features summing to one. The classes are displayed from bottom to top in order of increased intensity values, from the coronal holes $(\mathrm{Class} 1, \mathrm{CH})$ up to the brightest part of an active region (Class 6, AR3). The bottom panels show a zoom-in of the area coverage of the coronal holes. The data gaps in the EIT and SEM data are due to the SOHO vacation in summer and fall of 1998.

\section{Results}

Using synthetic spectra for each of the features, along with the area coverages derived from the SPoCA2 procedure described in the previous section, we determine the solar spectral irradiance $S$ as given in Eq. (2):

$$
S(\lambda, t)=\frac{1}{N(t)} \frac{\pi R_{\odot}^{2}}{D^{2}} \sum_{i, j} N_{j}\left(r_{i}, t\right) \cdot I_{j}\left(r_{i}, \lambda\right),
$$

where $D=1 \mathrm{AU}$ is the mean distance from the Earth to the Sun and $r_{i}$ are the center values of the concentric rings in relative solar radii for which the intensity spectrum $I_{i}$ is calculated. $N(t)$ is the total number of analyzed pixels of an image observed at time $t$ and $N_{j}\left(r_{i}, t\right)$ are the number of pixels associated with feature $j$ identified in ring $r_{i}$. We sum over features $j=1, \ldots, 6$ and rings $r_{i}$ from disk center to $1.35 R_{\odot}$. Figure 7 displays the 16 rings $r_{i}$ used in this reconstruction on a segmentation map produced by attribution. The class centers derived from the SOLMOD code are used; see Table 2. Generally, Eq. (2) is valid for any wavelength range. The suitability of the analyzed solar images and thus, the area coverage $N_{j}$ determined from them, depends on the question whether the chosen wavelengths are a good representation for the wavelength range under consideration.

Figure 8 shows the reconstruction based on the segmentation of the complete EIT dataset of $171 \AA$ and $195 \AA$ images using the same attribution procedure. The resulting EUV reconstruction between 26 and $34 \mathrm{~nm}$ is plotted in Figure 8 .
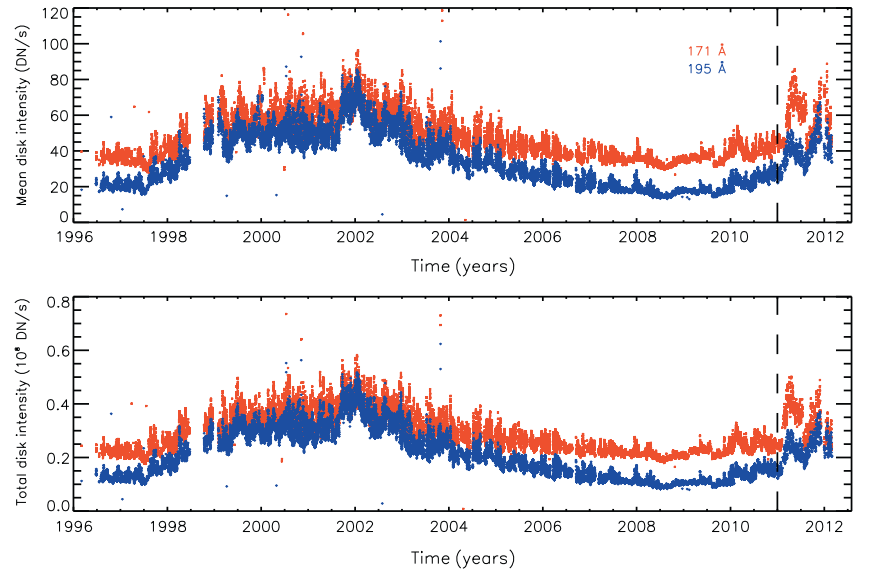

Fig. 6. Mean (top panel) and total (bottom panel) of the daily ondisk pixel intensities of the $171 \AA$ (red) and $195 \AA$ (blue) EIT images. Early in 2011 (indicated with the vertical dashed line), the time series shows a significant increase in mean and total intensity for both channels. Therefore, EIT data taken after 31 December 2010 have been excluded from the reconstruction.

The daily reconstruction (gray) and the 81-day mean (black) is compared to the SOHO/SEM data (light blue) and the 81-day mean (dark blue). Note that the data gaps in the $\mathrm{SOHO} / \mathrm{SEM}$ data as well as in the reconstruction are due to the SOHO vacation in summer and fall of 1998.

The best reconstruction, without any additional scaling, is obtained by using the area coverages for the coronal holes - as this 

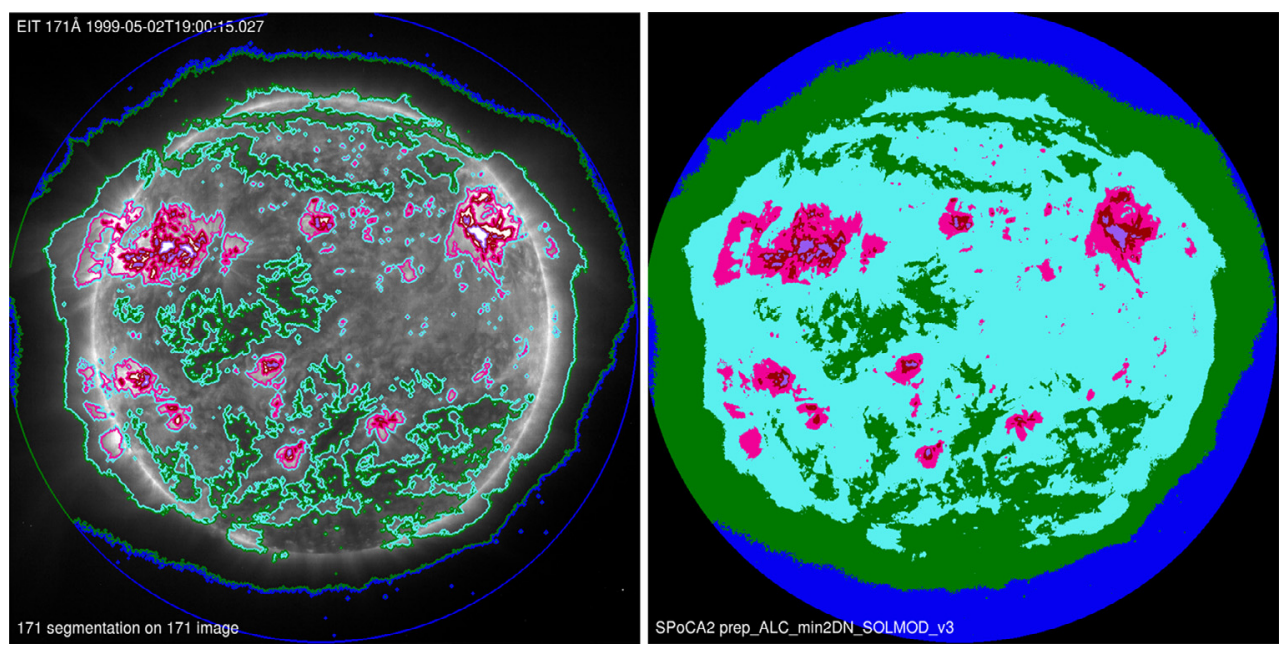

Fig. 7. The left panel shows the segmentation as an overlay on the original EIT $171 \AA$ image recorded on 2 May 1999 at 19:00:15. The right panel shows the segmentation map produced with the center values derived from SOLMOD.

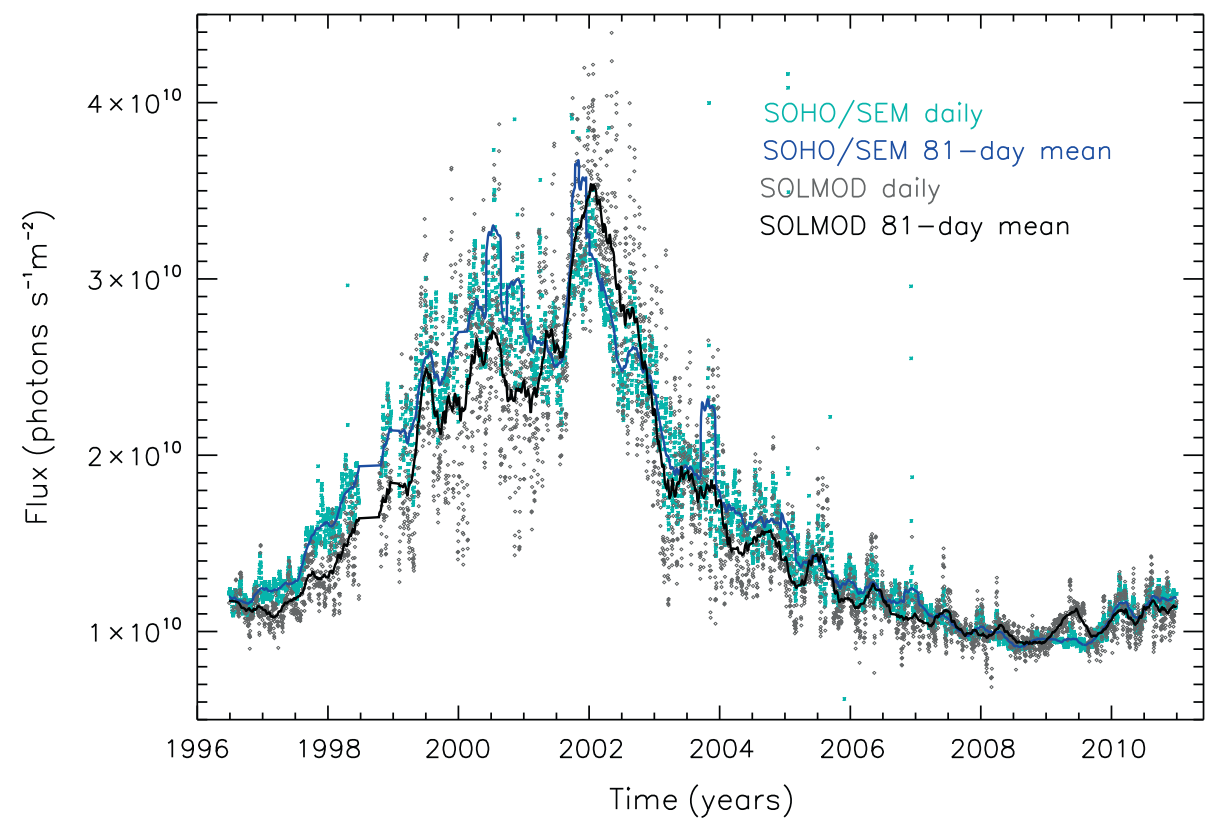

Fig. 8. Comparison of the SOLMOD reconstruction (gray) with the SOHO/SEM (light blue) irradiance data. The 81-day mean is also given for the reconstruction (black line) and the SOHO/SEM data (dark blue line). The data gaps in the SEM data as well as in the reconstruction are during the SOHO vacation in summer and fall of 1998.

channel is best suited for the identification of coronal holes and the active region classes AR1, AR2, and AR3 as derived from the $195 \AA$ channel. The remaining quiet-Sun classes QS1 and QS2 are determined from the $171 \AA$ channel. To meet the solar cycle variation and, most importantly, the solar minimum irradiance level, the QS1 class had to be represented in equal parts by the QS1 and QS2 spectrum. Furthermore, the active network class (AR1) is represented again in equal parts by the spectrum of the AR2 and AR3 class. A reason for the fact that the theoretical spectra underestimate the true intensity level is that the EIT images are not stray-light corrected. This means that dark features are contaminated by scattered light from the bright features. Moreover, for the reconstruction presented here, the two brightest features (AR2 and AR3) are represented by the AR3 spectrum. A possible reason for the missing variability might be that the position-dependent contribution of the background variability from the chromosphere and transition region is not taken into account in this study. Also, the intensity intervals for each of the classes, in particular, the active region classes (AR1 to AR3), is represented by one mean intensity for each class which can lead to an underestimation of the variability.

Finally, Figure 9 shows a scatter plot of the absolute variation of the SOHO/SEM data versus the variation of the SOLMOD reconstruction. The solid line indicates the second-order polynomial fit. The linear part of this fit is also indicated as dashed line with a correlation coefficient $r^{2}=0.82$. It can be concluded that the reconstruction reproduces the observed irradiance well at moderate solar activity. However, nonlinear effects come into play at high solar activity 


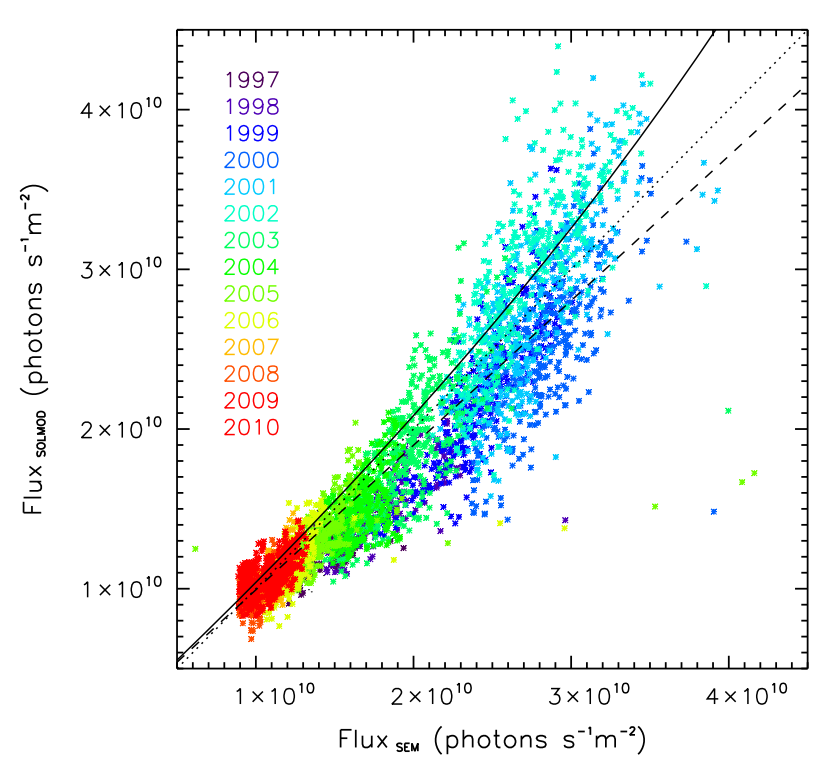

Fig. 9. Regression analysis of the SOLMOD reconstruction versus the SOHO/SEM data. The colors indicate the data point for the calendar years throughout the time series. The solid black line shows the second-order polynomial fit, and the dashed line gives the linear component of the fit with a correlation coefficient $r^{2}=$ 0.82 . The dotted line indicates the diagonal for better guidance.

which can be associated with very bright active regions, solar flares and energetic particles, as discussed below.

\section{Discussion}

The reconstruction of the EUV spectral irradiance for the $\mathrm{SOHO} / \mathrm{SEM}$ spectral band has been presented for more than one solar cycle. The overall agreement is reasonable, however some points need further investigation. First, the scatter of the reconstructed time series is larger than in the observations. The large scatter might be decreased by using the mean of more images per day for the daily mean irradiance value. Second, at times of solar maximum, the reconstruction clearly underestimates the observed variation. For the 2008 solar minimum, the reconstruction is slightly above the observations. One reason for this could be that the scattered light in the EIT instruments causes the fainter features to appear brighter, and even brighter features lose some intensity. Also, as the effect of flares is not taken into account in the SOLMOD reconstruction, the increase in EUV irradiance during the onset of a flare cannot be reproduced by the model. This can clearly explain some of the underestimation of the SOLMOD reconstruction.

Another important point is that, as shown by Bochsler et al. (2014) and Didkovsky et al. (2007), the signal in the SEM instrument increases during solar energetic particle events. Energetic particles can contribute significantly to the first-order SEM signal, increasing it by a factor of two or more in exceptional cases. Such contamination might explain some differences between the SEM observations and SOLMOD reconstruction, particularly the sharp increase in October/ November 2003 (which appears broadened over in the 81-day running mean) that occurred in the SEM data following strong particle events. However, as these energetic particle events are fairly isolated and short-lived, they can most likely not explain some of the more moderate but longer-term differences that appear throughout the rise of solar cycle 23 . The reason for these differences could probably be that, besides flares, very bright active regions appeared on the solar disk, which are underestimated by the hottest coronal model (AR3) used in SOLMOD. This indicates that besides a flare model, an additional hot coronal model should be developed.

The next steps will be to apply the reconstruction to other EUV image data sets such as e.g. PROBA2/SWAP and compare the results with PROBA2/LYRA (Hochedez et al. 2006; Kretzschmar et al. 2012; Dominique et al. 2013), ISS/ SOLAR/SolACES, SDO/EVE, and other available EUV irradiance measurements. Applying the same method to other spectral ranges will provide further constraints and validate our model for these spectral ranges.

\section{Conclusions}

We have presented a reconstruction of the solar EUV irradiance for the spectral range of 26-34 nm. A six-component model has been applied which accounts for coronal holes, quiet corona, coronal quiet network, coronal active network, active regions, and bright active regions. For the reconstruction of solar variations over the solar cycle we employed synthetic intensity spectra for different features. The comparison of the reconstruction with $\mathrm{SOHO} / \mathrm{SEM}$ data shows that the overall solar cycle variation is captured very well. However, some open issues, such as the underestimation of very hot active regions needs to be addressed in future work. Also, the effect of energetic particles on the detectors needs to be better understood and taken into account.

The work presented here is part of the FP7 SPACE Project SOLID with the aim to provide a homogeneous solar spectral irradiance dataset for the space era and beyond. As such it will be incorporated in an upcoming SSI composite for which new techniques are currently being developed within the FP7 SOLID Project.

Acknowledgements. We thank Tom Woods, Andrew Jones, and Peter Bochsler for discussions on EUV and specifically the SEM data, as well as Frédéric Auchère for helpful clarifications regarding the EIT data. Also, we thank Wilnelia Adams for carefully reading the manuscript. The editor and authors thank Frank Eparvier and Jeff Morrill for their assistance in evaluating this paper and very valuable comments. SOHO is a project of international cooperation between ESA and NASA. The research leading to these results has received funding from the European Community's Seventh Framework Programme (FP7/2007-2013) under Grant Agreement $\mathrm{N}^{\circ} 313188$ (SOLID, http://projects.pmodwrc.ch/solid/) and under the Grant Agreement $\mathrm{N}^{\circ} 263506$ (AFFECTS, http://www.affectsfp7.eu). Funding of BM, VD, and CV by the Belgian Federal Science Policy Office (BELSPO) through the ESA/PRODEX Telescience and SIDC Exploitation programs is also hereby acknowledged. This work was also supported by the SolarTerrestrial Centre of Excellence, a collaborative framework funded by the Belgian Science Policy Office and the COST Action ES1005 TOSCA (www.tosca-cost.eu).

\section{References}

Barra, V., V. Delouille, and J.-F. Hochedez, Segmentation of extreme ultraviolet solar images via multichannel fuzzy clustering, $A d v$. Space Res., 42, 917-925, DOI: 10.1016/j.asr.2007.10.021, 2008.

BenMoussa, A., S. Gissot, U. Schühle, G. Del Zanna, F. Auchère, et al., On-orbit degradation of solar instruments, Sol. Phys., 288, 389-434, DOI: 10.1007/s11207-013-0290-z, 2013. 
Bezdek, J., Pattern recognition with fuzzy objective function algorithms, Plenum Press, New-York, 1981.

Bochsler, P., H. Kucharek, E. Möbius, M. Bzowski, J.M. Sokół, L. Didkovsky, and S. Wieman, Solar photoionization rates for interstellar neutrals in the inner heliosphere: $\mathrm{H}, \mathrm{He}, \mathrm{O}$, and $\mathrm{Ne}$, Astrophys. J. Suppl. Ser., 210, 12 , DOI: 10.1088/0067-0049/210/1/12, 2014.

Cessateur, G., T. Dudok de Wit, M. Kretzschmar, J. Lilensten, J.-F. Hochedez, and M. Snow, Monitoring the solar UV irradiance spectrum from the observation of a few passbands, $A \& A, \mathbf{5 2 8}$, A68, DOI: 10.1051/0004-6361/201015903, 2011.

Cessateur, G., J. Lilensten, T. Dudok de Wit, A. BenMoussa, and M. Kretzschmar, New observation strategies for the solar UV spectral irradiance, J. Space Weather Space Clim., 2, A16, DOI: 10.1051/swsc/2012016, 2012.

Clette, F., J.-F. Hochedez, J.S. Newmark, J.D. Moses, F. Auchère, J.-M. Defise, and J.-P. Delaboudinière, The radiometric calibration of the extreme ultraviolet imaging telescope, ISSI Scientific Reports Series, 2, 121, 2002.

Delaboudinière, J.-P., G.E. Artzner, J. Brunaud, A.H. Gabriel, J.F. Hochedez, et al., EIT: Extreme-ultraviolet Imaging Telescope for the SOHO mission, Sol. Phys., 162, 291-312, DOI: 10.1007/BF00733432, 1995.

Dere, K.P., E. Landi, H.E. Mason, B.C. Monsignori Fossi, and P.R. Young, CHIANTI - an atomic database for emission lines, Astron. Astrophys. Suppl. Ser., 125, 149-173, DOI: 10.1051/aas:1997368, 1997.

Didkovsky, L.V., D.L. Judge, A.R. Jones, S. Wieman, and B.T. Tsurutani, Correction of SOHO CELIAS/SEM EUV measurements saturated by extreme solar flare events, Astron. Nachr., 328, 36-40, DOI: 10.1002/asna.200610667, 2007.

Didkovsky, L.V., D.L. Judge, S.R. Wieman, and D. McMullin. Minima of solar cycles $22 / 23$ and $23 / 24$ as seen in SOHO/CELIAS/SEM absolute solar EUV flux. In: S.R., Cranmer, J.T. Hoeksema, and J.L. Kohl, Editors, Astronomical Society of the Pacific Conference Series, 428, 73, 2010.

Dominique, M., J.-F. Hochedez, W. Schmutz, I.E. Dammasch, A.I. Shapiro, M. Kretzschmar, A.N. Zhukov, D. Gillotay, Y. Stockman, and A. BenMoussa, The LYRA instrument onboard PROBA2: description and in-flight performance, Sol. Phys., 286, 21-42, DOI: 10.1007/s11207-013-0252-5, 2013.

Dudok de Wit, T., and S. Bruinsma, Determination of the most pertinent EUV proxy for use in thermosphere modeling, Geophys. Res. Lett., 38, L19102,

DOI: 10.1029/2011GL049028, 2011.

Dudok de Wit, T., S. Bruinsma, and K. Shibasaki, Synoptic radio observations as proxies for upper atmosphere modelling, J. Space Weather Space Clim., 4, A06, DOI: 10.1051/swsc/2014003, 2014.

Fontenla, J.M., W. Curdt, M. Haberreiter, J. Harder, and H. Tian, Semiempirical models of the solar atmosphereIII. Set of non-LTE models for far-ultraviolet/extreme-ultraviolet irradiance computation, Astrophys. J., 707, 482-502, DOI: 10.1088/0004-637X/707/1/482, 2009.

Fontenla, J.M., J. Harder, W. Livingston, M. Snow, and T. Woods, High-resolution solar spectral irradiance from extreme ultraviolet to far infrared, J. Geophys. Res. (Atmos.), 116, D20108, DOI: 10.1029/2011JD016032, 2011.

Haberreiter, M., Solar EUV spectrum calculated for quiet sun conditions, Sol. Phys., 274, 473-479, DOI: $10.1007 / \mathrm{s} 11207-011-9767-9,2011$

Haberreiter, M.. Towards the reconstruction of the EUV irradiance for solar cycle 23. In: C.H., Mandrini, and D.F. Webb, Editors, IAU Symposium, 286, 97-100,

DOI: $10.1017 / \mathrm{S} 174392131200470 \mathrm{X}, 2012$.

Haberreiter, M., W. Schmutz, and A.G. Kosovichev, Solving the discrepancy between the seismic and photospheric solar radius, Astrophys. J., 675, L53-L56, DOI: 10.1086/529492, 2008.
Hinteregger, H.E., K. Fukui, and B.R. Gilson, Observational reference and model data on solar EUV, from measurements on AE-E, Geophys. Res. Lett., 8, 1147-1150, 1981.

Hochedez, J., W. Schmutz, Y. Stockman, U. Schühle, A. Benmoussa, et al., LYRA, a solar UV radiometer on Proba2, Adv. Space Res., 37, 303-312, DOI: 10.1016/j.asr.2005.10.041, 2006.

Hovestadt, D., M. Hilchenbach, A. Bürgi, B. Klecker, P. Laeverenz, et al., CELIAS - Charge, Element and Isotope Analysis System for SOHO, Sol. Phys., 162, 441-481, DOI: 10.1007/BF00733436, 1995.

Judge, D.L., D.R. McMullin, H.S. Ogawa, D. Hovestadt, B. Klecker, et al., First solar EUV irradiances obtained from SOHO by the CELIAS/SEM, Sol. Phys., 177, 161-173, DOI: 10.1023/A:1004929011427, 1998.

Kretzschmar, M., I.E. Dammasch, M. Dominique, J. Zender, G. Cessateur, and E. D'Huys, Extreme ultraviolet solar irradiance during the rising phase of solar cycle 24 observed by PROBA2/ LYRA, J. Space Weather Space Clim., 2, A14, DOI: 10.1051/swsc/2012014, 2012.

Kretzschmar, M., J. Lilensten, and J. Aboudarham, Retrieving the solar EUV spectral irradiance from the observation of 6 lines, Advances in Space Research, 37, 341-346, DOI: 10.1016/j.asr.2005.02.029, 2006.

Krishnapuram, R., and J. Keller, A possibilistic approach to clustering, IEEE Trans. Fuzzy Systems, 1, 98-110, 1993.

Krishnapuram, R., and J. Keller, The possibilistic C-means algorithm: Insights and recommendations, IEEE Trans. Fuzzy Systems, 4, 385-393, 1996.

Landi, E., G. Delzanna, P.R. Young, K.P. Dere, H.E. Mason, and M. Landini, CHIANTI-An atomic database for emission lines. VII. New data for X-rays and other improvements, Astrophys. $J$. Suppl. Ser., 162, 261-280, DOI: 10.1086/498148, 2006.

Laštovička, J., Are trends in total electron content (TEC) really positive, J. Geophys. Res. (Space Phys.), 118, 3831-3835, DOI: 10.1002/jgra.50261, 2013

Lean, J.L., J.T. Emmert, J.M. Picone, and R.R. Meier, Global and regional trends in ionospheric total electron content, J. Geophys. Res. (Space Phys.), 116, A00H04, DOI: 10.1029/2010JA016378, $2011 \mathrm{a}$.

Lean, J.L., T.N. Woods, F.G. Eparvier, R.R. Meier, D.J. Strickland, J.T. Correira, and J.S. Evans, Solar extreme ultraviolet irradiance: Present, past, and future, J. Geophys. Res. (Space Phys.), 116, A01102, DOI: 10.1029/2010JA015901, 2011b.

Lemen, J.R., A.M. Title, D.J. Akin, P.F. Boerner, C. Chou, et al., The Atmospheric Imaging Assembly (AIA) on the Solar Dynamics Observatory (SDO), Sol. Phys., 275, 17-40, DOI: 10.1007/s11207-011-9776-8, 2012.

Lilensten, J., T. Dudok de Wit, M. Kretzschmar, P.-O. Amblard, S. Moussaoui, J. Aboudarham, and F. Auchère, Review on the solar spectral variability in the EUV for space weather purposes, Ann. Geophys., 26, 269-279, 2008.

McMullin, D.R., D.L. Judge, M. Hilchenbach, F. Ipavich, P. Bochsler, P. Wurz, A. Burgi, W.T. Thompson, and J.S. Newmark, In-flight comparisons of solar EUV irradiance measurements provided by the CELIAS/SEM on SOHO, ISSI Scientific Reports Series, 2, 135, 2002.

Ogawa, H.S., D.R. McMullin, D.L. Judge, and R.S. Korde, Normal incidence spectrophotometer with high-density transmission grating technology and high-efficiency silicon photodiodes for absolute solar extreme-ultraviolet irradiance measurement, Opt. Eng., 32, 3121-3125, DOI: 10.1117/12.149195, 1993.

Qian, L., and S.C. Solomon, Thermospheric density: an overview of temporal and spatial variations, Space Sci. Res., 168, 147-173, DOI: 10.1007/s11214-011-9810-z, 2012.

Schmidtke, G., R. Brunner, D. Eberhard, B. Halford, U. Klocke, M. Knothe, W. Konz, W.-J. Riedel, and H. Wolf, SOL-ACES: Auto-calibrating EUV/UV spectrometers for measurements onboard the International Space Station, Adv. Space Res., 37, 273-282, DOI: 10.1016/j.asr.2005.01.112, 2006a. 
M. Haberreiter et al.: Reconstruction of the solar EUV irradiance from 1996 to 2010

Schmidtke, G., C. Fröhlich, and G. Thuillier, ISS-SOLAR: Total (TSI) and spectral (SSI) irradiance measurements, Adv. Space Res., 37, 255-264, DOI: 10.1016/j.asr.2005.01.009, 2006 b.

Thuillier, G., J. Claudel, D. Djafer, M. Haberreiter, N. Mein, S.M.L. Melo, W. Schmutz, A. Shapiro, C.I. Short, and S. Sofia, The shape of the solar limb: Models and observations, Sol. Phys., 268, 125-149, DOI: 10.1007/s11207-010-9664-7, 2011.

Thuillier, G., G. Schmidtke, C. Erhardt, B. Nikutowski, A.I. Shapiro, et al., Solar spectral irradiance variability in November/December 2012: Comparison of observations by instruments on the international space station and models, Sol. Phys., DOI: 10.1007/s11207-014-0588-5, 2014.

Verbeeck, C., V. Delouille, B. Mampaey, and R. De Visscher, The SPoCA-suite: Software for extraction, characterization, and tracking of active regions and coronal holes on EUV images, $A \& A$, 561, A29, DOI: 10.1051/0004-6361/201321243, 2014.

Warren, H.P., J.T. Mariska, and J. Lean, A new model of solar EUV irradiance variability: 1. Model formulation, J. Geophys. Res., 106, 15,745-15,758, DOI: 10.1029/2000JA000282, 2001.
Wieman, S.R., L.V. Didkovsky, and D.L. Judge, Resolving differences in absolute irradiance measurements between the SOHO/ CELIAS/SEM and the SDO/EVE, Sol. Phys., 289, 2907-2925, DOI: 10.1007/s11207-014-0519-5, 2014.

Wieman, S.R., D.L. Judge, and L.V. Didkovsky, Solar EUV Monitor (SEM) absolute irradiance measurements and how they are affected by choice of reference spectrum. Proc. SPIE, 8148, DOI: 10.1117/12.893163, 2011.

Woods, T.N., F.G. Eparvier, S.M. Bailey, P.C. Chamberlin, J. Lean, G.J. Rottman, S.C. Solomon, W.K. Tobiska, and D.L. Woodraska, Solar EUV Experiment (SEE): mission overview and first results, J. Geophys. Res. (Space Phys.), 110, A01312, DOI: 10.1029/2004JA010765, 2005.

Woods, T.N., F.G. Eparvier, R. Hock, A.R. Jones, D. Woodraska, et al., Extreme ultraviolet Variability Experiment (EVE) on the Solar Dynamics Observatory (SDO): Overview of science objectives, instrument design, data products, and model developments, Sol. Phys., 275, 115-143, DOI: $10.1007 / \mathrm{s} 11207-009-9487-6,2012$.

Cite this article as: Haberreiter M, Delouille V, Mampaey B, Verbeeck C, Del Zanna G, et al.: Reconstruction of the solar EUV irradiance from 1996 to 2010 based on SOHO/EIT images. J. Space Weather Space Clim., 2014, 4, A30. 